\title{
Time-Resolved Limited Proteolysis of Mitogen-Activated Protein Kinase-Activated Protein Kinase-2 Determined by LC/MS Only
}

\author{
Li Tao, ${ }^{\mathrm{a}, *}$ Susan E. Kiefer, ${ }^{\mathrm{a}}$ Dianlin Xie, ${ }^{\mathrm{a}}$ James W. Bryson, ${ }^{\mathrm{a}}$ \\ Stanley A. Hefta, ${ }^{b}$ and Michael L. Doyle ${ }^{a}$ \\ ${ }^{a}$ Department of Gene Expression and Protein Biochemistry, Bristol-Myers Squibb Research and \\ Development, Princeton, New Jersey, USA \\ ${ }^{\mathrm{b}}$ Department of Proteomics, Bristol-Myers Squibb Research and Development, Princeton, New Jersey, USA
}

\begin{abstract}
Mass spectrometry has gained prominence in limited proteolysis studies largely due to its unparalleled precision in determining protein molecular mass. However, proteolytic fragments usually cannot be identified through direct mass measurement, since multiple subsequences of a protein can frequently be matched to observed masses of proteolytic fragments. Therefore, additional information from N-terminal sequencing is often needed. Here we demonstrate that mass spectrometry analysis of the time course of limited proteolysis reactions provides new information that is self-sufficient to identify all proteolytic fragments. The method uses a non-specific protease like subtilisin and exploits information contained in the time-resolved dataset such as: increased likelihood of identifying larger fragments generated during initial proteolysis solely by their masses, additivity of the masses of two mutually exclusive sequence regions that generate the full-length molecule (or an already assigned subfragment), and analyses of the proteolytic subfragment patterns that are facilitated by having established the initial cleavage sites. We show that the identities of the observed proteolytic fragments can be determined by LC/MS alone because enough constraints exist in the time-resolved dataset. For a medium-sized protein, it takes about $8 \mathrm{~h}$ to complete the study, a significant improvement over the traditional SDS-PAGE and N-terminal sequencing method, which usually takes several days. We illustrate this method with application to the catalytic domain of mitogen-activated protein kinase-activated protein kinase-2, and compare the results with $\mathrm{N}$-terminal sequencing data and the known X-ray crystal structure. (J Am Soc Mass Spectrom 2008, 19, 841-854) (c 2008 American Society for Mass Spectrometry
\end{abstract}

L imited proteolysis is a method that has been used for decades in a wide range of applications involving protein structure analysis. The method consists of two parts: proteolysis of the target protein under controlled conditions where the protein is only partially digested, followed by identification of protease-resistant stable products. The first part is well established and has remained essentially unchanged methodologically over the years, even though the precise reason for why some sites are sensitive to cleavage, whereas others are resistant, is not entirely known. Nevertheless, it is generally accepted that accessible, flexible, and unstructured regions are preferably cleaved [1,2] and that stably folded and structured fragments are more resistant to cleavage. The second part of the method, identifying limited proteolysis

Address reprint requests to Dr. Li Tao, Department of Protein Therapeutics Development, Bristol-Myers Squibb R\&D, PO Box 5400, Princeton, NJ 08534. E-mail: li.tao@bms.com.

Readers can also contact Dr. Michael L. Doyle, Department of Gene Expression and Protein Biochemistry, Bristol-Myers Squibb R\&D, PO Box 4000, Princeton, NJ 08543. E-mail: michael.doyle@bms.com

${ }^{*}$ Current organization: Department of Protein Therapeutics Development, Research and Development, Bristol-Myers Squibb, Princeton, NJ 08534. products, has traditionally been done by SDS-PAGE coupled with N-terminal sequencing. However, this approach is slow, tedious, and usually cannot determine the C-termini of proteolysis products due to insufficient accuracy of SDS-PAGE for measuring protein fragment mass.

Advances in matrix-assisted laser desorption/ionization time-of-flight mass spectrometry (MALDITOFMS or MALDI) and high performance liquid chromatography with electrospray ionization mass spectrometry (HPLC-ESI-MS or LC/MS) have revolutionized mass spectral analysis of macromolecules with greater mass accuracy and speed. As a result, much more precise information about proteolysis products can be obtained when mass spectrometry is used. In the past ten years or so, many studies involving limited proteolysis have used mass spectrometry, such as binding epitope mapping [3-7], domain structure and function [8-14], protein-protein interaction and assembly [15-18], protein modification [19-21], protein conformation and topology [22-27], and domain isolation and crystallography [28-30], for example. Generally speaking, unless specific enzymes are used, MS-based ap- 


\title{
1 MQQFPQFHVK SGLQIKKNAI IDDYKVTSQV LGLGINGKVL QIFNKRTQEK
}

51 FALKMLQDCP KARREVELHW RASQCPHIVR IVDVYENLYA GRKCLLIVME

101 CLDGGELFSR IQDRGDQAFT EREASEIMKS IGEAIQYLHS INIAHRDVKP

201 YDKSCDMWSL GVIMYILLCG YPPFYSNHGL *ैAISPGMKTRI RMGQYEFPNP

251 EWSEVSEEVK MLIRNLLKTE PTQRMTITEF MNHPWIMQST KVPQTPLHTS

\section{RVLKEDKERW EDVKEEMTSA LATMR}

\begin{abstract}
Scheme 1. Protein sequence of the catalytic domain of the mitogen-activated protein kinaseactivated protein kinase-2 (MK2) construct used in this study (calculated mass $=37,601.5 \mathrm{Da}$ ). The subtilisin cleavage sites identified in the present study are marked by arrows and asterisks. Arrows indicate sites that are the most susceptible to subtilisin.
\end{abstract}

proaches still need additional information from other techniques such as N-terminal sequencing to unambiguously identify proteolysis products. This is because many fragments of a protein molecule can have very similar masses that are too close to be differentiated by a simple mass spectrometric measurement. A common way to bypass this hurdle is using high-specificity proteases such as trypsin to cleave at specific residues. Although this approach facilitates assignment of detected masses to fragments, it limits the analysis to regions of the target protein that contain the preferred amino acid sequence. In addition, many so-called specific proteases will cleave both primary recognition sequences and secondary sequences, thus further complicating identification of fragments. Alternatively, peptide mapping on HPLC isolated fractions or SDS-PAGE separated bands could provide additional information on sequence coverage that could in principle facilitate fragment identification. However, each HPLC fraction or gel band requires significant amounts of sample preparation and enzymatic digestion time (hours) followed by additional time for LC/MS/MS analysis. A complete limited proteolysis study by peptide mapping could take several days to finish. In addition, it can sometimes be challenging to determine the $\mathrm{N}$ - or $\mathrm{C}$ termini of protein fragments by peptide mapping.

Recently Stroh and colleagues [31] reported a method for using mass spectrometry alone to identify the $\mathrm{N}$ - and C-termini of stable fragments generated by limited proteolysis with semispecific or non-specific proteases. They showed that limited proteolysis products can be identified in cases where semispecific or non-specific proteases generate closely related fragments containing the same $\mathrm{N}$ - or $\mathrm{C}$-terminus on one end while differing by a few residues at the other end. The Stroh studies prompted us to develop a general method for analyzing limited proteolysis products that solely relies on $\mathrm{LC} / \mathrm{MS}$.

Here, we report a new, time-resolved LC/MS method for identifying proteolytic fragments of target protein molecules using a non-specific protease such as subtilisin. The approach exploits mass and sequence constraints to eliminate false sequences matched to observed masses of proteolytic fragments. The most useful constraints are sequence exclusivity, sequence and mass complementarities (masses and sequence regions that sum to that of the intact protein), sequence and mass similarities of fragments co-eluting in LC separation, and the connectivity that exists between the known initial cleavage sites and the chronological pattern of sub-fragmentation that proceeds until the reaction is stopped. The time-resolved approach enables a more rapid and information-rich means for conducting structural analyses of protein targets by limited proteolysis.

\section{Experimental}

\section{Protein Reagents}

The DNA coding region for a truncated form (residues 41-364, as shown in Scheme 1) of isoform 2 of mitogenactivated protein kinase-activated protein kinase-2 (MAPKAP-K2 or MK2, RefSeq \#NP116584) was cloned into a modified version of the commercially available pET28b vector (Novagen) in which the multiple-cloning site had been rearranged and the N-terminal His-tag region removed. 
The resulting expressed protein entails MK2 isoform (241-364) with a single Met residue added on the $\mathrm{N}$-terminus. The construct was expressed in E. coli BL21(DE3) cells (Novagen) in M9 minimal media supplemented with casamino acids and trace minerals. The recombinant protein was purified by cation exchange chromatography using an SP-Sepharose Fast Flow column, followed by size-exclusion chromatography using a Superdex 200 column, and yielded a product with a purity of $>98 \%$, as assessed by SDS-PAGE. Purified MK2 was stored at $-80{ }^{\circ} \mathrm{C}$ in small aliquots, at a concentration of $1 \mathrm{mg} / \mathrm{mL}$ in $20 \mathrm{mM}$ HEPES, $\mathrm{pH} 7.5$, containing $0.2 \mathrm{M} \mathrm{NaCl}, 1 \mathrm{mM}$ EDTA, and $10 \mathrm{mM}$ DTT. A fresh aliquot was thawed and used for each experiment.

Subtilisin Carlsberg was purchased from SigmaAldrich (St. Louis, MO, USA) as lyophilized powder (Cat. \#P5380), reconstituted in $10 \mathrm{mM}$ Tris, $\mathrm{pH} 8.0$, containing $150 \mathrm{mM} \mathrm{NaCl}$, to $1 \mathrm{mg} / \mathrm{mL}$, at $0{ }^{\circ} \mathrm{C}$, and immediately frozen at $-80^{\circ} \mathrm{C}$ in small aliquots. A fresh aliquot was used in each experiment.

\section{Enzyme-to-Substrate Ratio}

An appropriate enzyme-to-substrate ratio is needed to ensure that sufficient proteolysis occurs to allow stable domains to be identified, but not so much that the target protein is overly digested. When subtilisin was used as the protease, we have found an enzyme-to-substrate $(\mathrm{E} / \mathrm{S})$ ratio of $1 / 2000(\mathrm{~m} / \mathrm{m})$ is optimal for most proteins (data not shown). This ratio usually allows all major proteolysis events to be captured over the 6-h time course. If the starting ratio was not suitable for a target protein, depending on the situation, increasing or decreasing the ratio by 10 -fold would usually result in an acceptable digestion.

\section{Limited Proteolysis}

At time zero, MK2 at a concentration of $1.0 \mathrm{mg} / \mathrm{mL}$ was mixed with subtilisin at a mass ratio of $E / S=1 / 20,000$. The sample was then placed in an autosampler that injected the reaction mixture into the LC/MS system at different time points. The sample tray of the autosampler was maintained at $25 \pm 0.3^{\circ} \mathrm{C}$. The first injection of $5 \mu \mathrm{L}$ of reaction mixture was made 2 min after MK2 was mixed with subtilisin. Additional injections of reaction mixture in equal volume were made every $15 \mathrm{~min}$ thereafter for the first $2 \mathrm{~h}$, then every $30 \mathrm{~min}$ for the following $4 \mathrm{~h}$. In the time course experiment using the traditional SDS-PAGE and N-terminal sequencing method, aliquots of $10 \mu \mathrm{L}$ sample were collected at various time points, and $1 \mathrm{mM}$ phenylmethanesulfonyl fluoride (Cat. \#P7626, Sigma-Aldrich) was added to stop the reaction before each tube was placed on dry ice. After all time point samples were collected, the fractions were thawed, mixed with lithium dodecyl sulfate sample buffer (Cat. \#NP0007, Invitrogen, Carlsbad, CA, USA), and loaded directly onto a $4-12 \%$ NuPage gel
(Invitrogen) running in MES [2-(N-morpholino) ethanesulfonic acid] buffer.

\section{LC/MS Analysis}

The HPLC system including a temperature-controlled autosampler, an HPLC pump, and a photodiode detector, was made by Thermo Fisher Scientific (Waltham, MA, USA). The reversed-phase liquid chromatography (RPLC) column (Jupiter $5 \mu$ C4 $300 \AA$, $50 \times 2.0 \mathrm{~mm}$ ), used for separating proteolytic fragments, was purchased from Phenomenex (Torrance, CA, USA). The mobile phases were $0.05 \%$ trifluoroacetic acid (TFA; Fluka, Cat. \#91,707) dissolved in Millipore water (A) and acetonitrile (B) (Cat. \#10071618, Honeywell Burdick \& Jackson, Muskegon, MI, USA). The separation was done at a flow rate of $200 \mu \mathrm{L} / \mathrm{min}$ with a combination of several linear gradients. For 15-min separation, the gradient in percentage of A was 100-57, 57-52, 52-100, $100-$, in $1,8,1$, and $5 \mathrm{~min}$, respectively. For 30-min separation, the gradient in percentage of A was 100-60, $60-57,57-53,53-44,44-30,30-100,100-$, in 4, 5, 5, 5, 3, 2 , and $6 \mathrm{~min}$, respectively. Eluted protein fragments from the RPLC column were fed into an LCQ-DUO ion trap mass spectrometer (Thermo Fisher Scientific) through a sample-introducing capillary heated at $200{ }^{\circ} \mathrm{C}$. Raw data collected on each mass spectrum were averaged using the Boxcar smoothing algorithm with five data points. Preliminary mass spectra were first obtained by deconvoluting raw data using ProMass (Novatia LLC, Princeton, NJ, USA), a software program for automatic deconvolution of multiply charged macromolecular ions. The major masses obtained from ProMass analysis were then further analyzed by Bioworks 3.2 (Thermo Fisher Scientific) using the Biomass Calculation and Deconvolution function to obtain final mass spectra.

\section{Mass Recalibration}

We have observed that the main source of error in mass measurement by our ion trap mass spectrometers is instrumental drift. We have also found this effect can easily be rectified by adjusting measured masses with a linear recalibration curve. The recalibration curve was obtained by measuring masses of three control proteins (masses ranging from about 10 to $60 \mathrm{kDa}$ ) and correlating measured masses with their respective calculated masses.

\section{Results and Discussion}

The time-resolved limited proteolysis method we have developed consists of two general procedures. The first involves experimental generation of limited proteolysis fragments at appropriate time intervals and over an appropriate total time period, followed by chromatographic separation of the proteolytic fragments and determination of their masses by mass spectrometry. 


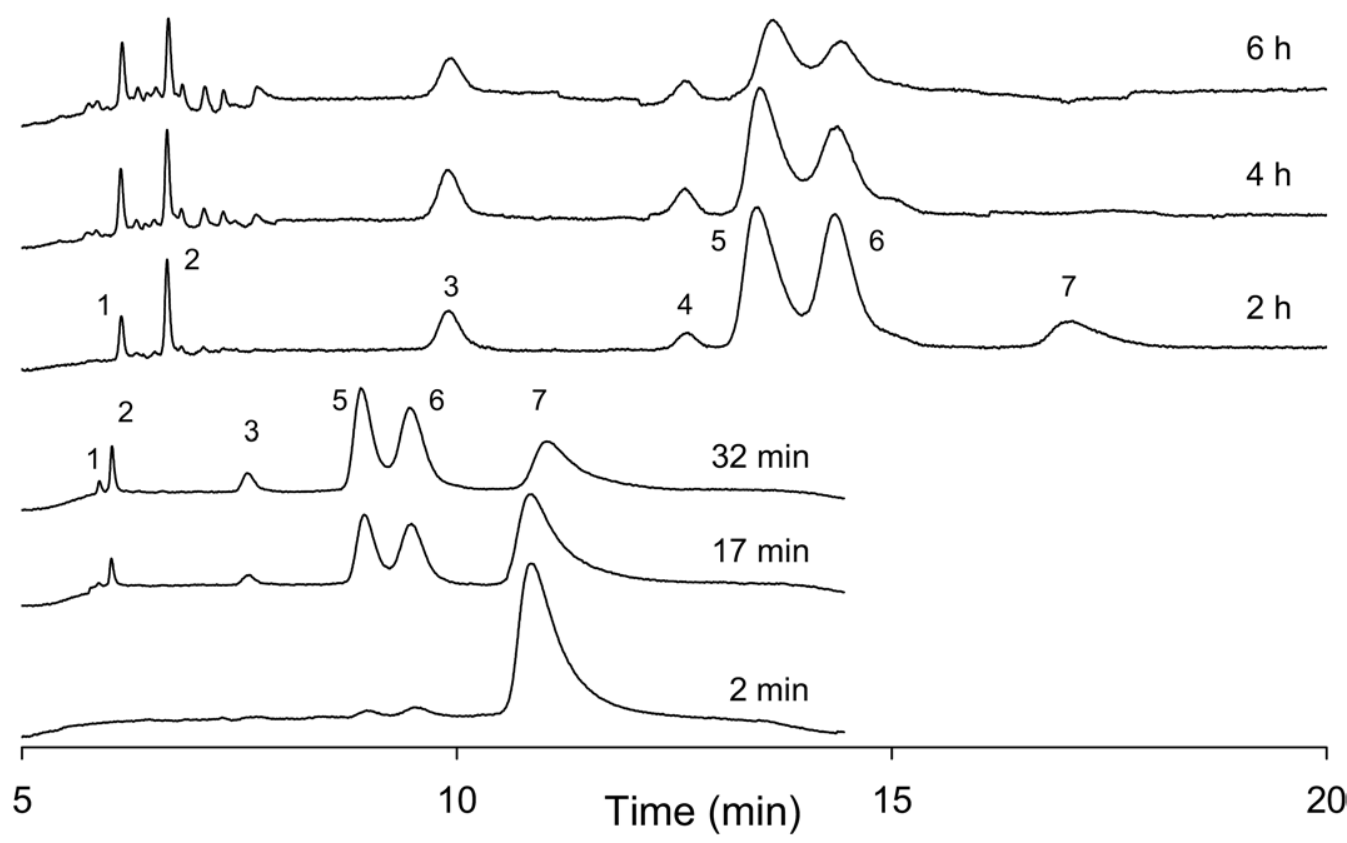

Figure 1. Chromatographic profiles of proteolytic fragments measured by UV absorption at $214 \mathrm{~nm}$ at labeled time points. MK2 was mixed with subtilisin at time zero. The reaction was carried out at $25^{\circ} \mathrm{C}$ for $6 \mathrm{~h}$ (bottom to top). Aliquots of the reaction mixture were sampled and analyzed by LC/MS $2 \mathrm{~min}$ after the reaction started, and every $15 \mathrm{~min}$ for the next $2 \mathrm{~h}$, followed by every $30 \mathrm{~min}$ for the next $4 \mathrm{~h}$, using two different gradients (top 3 versus bottom 3 profiles) as described in the Experimental section. Equivalent peaks for the two gradients are indicated by the numbers. Mass spectra of all chromatographic peaks (except the unlabeled minor ones between peak $\mathbf{1}$ and peak $\mathbf{3}$ ) are summarized in Table 1.

The second involves interpretation of all the data generated throughout the time course in a way that leads to a self-consistent set of constraints that enable determination of the identities of proteolytic fragments, including intermediates.

\section{LC Separation of Fragments and Determination of Masses}

Shown in Figure 1 are six chromatograms from timeresolved limited proteolysis of $\mathrm{MK} 2$, in which major peaks were denoted as peak 1 through peak 7 . As can be seen from the figure, the proteolysis products in peaks 5 and $\mathbf{6}$ were visible at very early stages of the reaction. The amount of intact MK2 (in peak 7) decreased over time and became undetectable by the 4 -h time point. A total of at least six major proteolysis products (from peak 1 through peak 6 ) were formed and remain visible by the 6-h time point. Mass spectral analysis on each chromatographic peak over the entire time course detected a total of 12 species, as shown in Table 1 . The blank fields in Table 1 are those at which a fragment was not detected or the signals were too weak to have a dependable measurement. Average mass values for each fragment are also given in the table. Averaging the measured mass over several time points was performed to reduce random error.

\section{Generation of the Time Dependence of Proteolytic Fragments}

In this study, the proteolysis products were well separated, given that only peaks 5, 6, and 7 contained more than one fragment. It was possible to obtain a dynamic, semiquantitative description of all proteolysis products by plotting their quantities, obtained from the LC profile, versus time as shown in Figure 2. For most HPLC peaks (measured by UV absorption at $214 \mathrm{~nm}$ ) containing only one fragment, the peak height was used directly to quantify a fragment's total amount. If more than one fragment were present in the HPLC peak, each fragment's quantity was approximated based on the assumption that those very similar fragments have similar ionization potentials; thus their mass spectral intensities were proportional to their relative quantities. Although the identities of the fragments are still unknown before further analysis, their relative stabilities were distinctively different based on the profiles shown in Figure 2. It is very easy to differentiate stable fragments (the top 7 curves with solid lines) from those transient species (the bottom 5 curves with dashed lines). Based on stability, fragments are categorized into three groups: the most stable ones (denoted by "ms"), the relatively stable ones (denoted by "rs"), and the transient ones (denoted by " $\operatorname{tr}^{\prime \prime}$ ). The quantities of the most stable fragments did not show an obvious de- 
Table 1. Detected fragment masses of MK2 from each chromatographic peak at different time points. ${ }^{\text {a }}$

\begin{tabular}{|c|c|c|c|c|c|c|c|c|c|c|c|c|}
\hline $\begin{array}{l}\text { Time } \\
\text { point } \\
\text { (min) }\end{array}$ & & & & & Detecte & fragmen & masses & Da) & & & & \\
\hline 2 & 37602 & & 21703 & 20777 & & & 16842 & 15916 & & & & \\
\hline 17 & 37600 & 36330 & 21705 & 20777 & 20431 & 19508 & 16843 & 15917 & 11265 & & 1288.72 & \\
\hline 32 & 37601 & 36328 & 21704 & 20778 & 20432 & 19506 & 16842 & 15917 & 11265 & & 1288.66 & \\
\hline 47 & 37602 & 36329 & 21704 & 20779 & 20433 & 19505 & 16843 & 15916 & 11264 & & 1288.72 & 944.32 \\
\hline 62 & 37601 & 36331 & 21706 & 20779 & 20433 & 19504 & 16845 & 15917 & 11265 & & 1288.72 & 944.32 \\
\hline 77 & 37602 & 36330 & 21704 & 20777 & 20433 & 19506 & 16843 & 15917 & 11264 & & 1288.72 & 944.39 \\
\hline 92 & 37602 & 36331 & 21703 & 20778 & 20434 & 19507 & & 15917 & 11265 & & 1288.72 & 944.39 \\
\hline 107 & 37601 & 36331 & 21706 & 20777 & 20432 & 19506 & & 15917 & 11265 & & 1288.72 & 944.32 \\
\hline 122 & 37601 & 36330 & 21703 & 20777 & 20433 & 19506 & & 15917 & 11265 & & 1288.66 & 944.39 \\
\hline 152 & & 36330 & 21704 & 20777 & 20432 & 19506 & & 15917 & 11264 & 4670 & 1288.72 & 944.39 \\
\hline 182 & & 36330 & 21703 & 20777 & 20433 & 19506 & & 15916 & 11265 & 4670 & 1288.72 & 944.39 \\
\hline 212 & & 36328 & & & 20432 & 19506 & & 15916 & 11264 & 4670 & 1288.72 & 944.39 \\
\hline 242 & & 36328 & & & 20432 & 19506 & & 15916 & 11264 & 4670 & 1288.72 & 944.39 \\
\hline 272 & & & & & 20432 & 19506 & & 15916 & 11264 & 4670 & 1288.72 & 944.39 \\
\hline 302 & & & & & 20433 & 19506 & & 15917 & 11264 & 4670 & 1288.79 & 944.39 \\
\hline 332 & & & & & 20433 & 19505 & & 15916 & 11264 & 4670 & 1288.66 & 944.39 \\
\hline 362 & & & & & 20432 & 19505 & & 15916 & 11264 & 4670 & 1288.72 & 944.39 \\
\hline Average & 37601.3 & 36329.9 & 21704.1 & 20777.3 & 20432.5 & 19506.0 & 16843.0 & 15916.8 & 11264.7 & 4670.0 & 1288.71 & 944.38 \\
\hline SD & \pm 0.7 & \pm 1.1 & \pm 1.1 & \pm 1.1 & \pm 0.7 & \pm 0.9 & \pm 1.2 & \pm 0.5 & \pm 0.5 & \pm 0.0 & \pm 0.03 & \pm 0.03 \\
\hline $\begin{array}{l}\text { HPLC } \\
\text { peak }\end{array}$ & p7 & p7 & p5 & p5 & p5 & p5 & p6 & p6 & p3 & $\mathrm{p} 4$ & $\mathrm{p} 2$ & $\mathrm{p} 1$ \\
\hline
\end{tabular}

Averaged masses and their corresponding standard deviations (SDs) are also given. The name of the HPLC peak in which each mass was detected is also shown.

${ }^{a}$ The blank fields are those at which certain fragments were not detected or the signals were too weak to yield reliable measurements.

crease during the time course. The quantities of relatively stable fragments did decrease over time, but significant amounts were still detected at the 6-h time point. The quantity of each transient fragment reached their maximum during the early phase of proteolysis (20-40 $\mathrm{min}$ from the start of the reaction), then decreased dramatically in the following $2 \mathrm{~h}$, and became undetectable by the 4 -h time point. In contrast, a significant amount of each stable fragment was still detected at the 6-h time point. We use a nomenclature for each fragment that starts with a two-letter code denoting the stability of the fragment, followed by the name of the chromatographic peak in which the fragment was detected, and its average mass. For example, the transient fragment detected in peak 7 with an average mass of $36,329.9 \mathrm{Da}$ is referred to as tr-p736,329.9 Da.

To summarize the time dependence of the fragments observed, the most stable fragments were: ms-p21288.71 Da, ms-p3-11,264.7 Da, and ms-p4-4670.0 Da; the relatively stable ones were: rs-p6-15,916.8 Da, rs-p519,506.0 Da, rs-p5-20,432.5 Da, and rs-p1-944.38 Da; the transient fragments including the intact MK2 molecule were: tr-p6-16,843.0 Da, tr-p5-20,777.3 Da, tr-p5-21,704.1 $\mathrm{Da}$, tr-p7-36,329.9 Da, and tr-p7-37,601.3 Da. Once these fragments are identified, this profile will provide a detailed description of the whole proteolysis process with all major reaction products including transient ones accounted for.

\section{Identification of Proteolytic Fragments from Observed Masses and Matched Sequences}

For each observed fragment, a list of subsequences of MK2 can be assembled whose calculated masses match, within error, that of the fragment. That is, if the observed fragments are treated as discrete variables, the lists corresponding to each observed fragment are their potential values. The theoretical basis of the current method is that if enough independent constraints exist, all variables that are subject to these constraints can be determined unambiguously. For this reason, the success of the current method depends on whether enough relationships among proteolytic fragments can be found. Time-resolved limited proteolysis provides a way to follow the proteolysis reaction as it proceeds and identify relationships among various fragments at different time stages of the reaction. As more constraints are identified during data analysis, more false sequences can be eliminated. Eventually, only one sequence remains on the list that is the true value or the identity of each fragment. Guidelines on how to approach the data analysis are subsequently described.

Similar to solving equations with multiple unknowns, multiple approaches exist in eliminating false sequences during data analysis. Nevertheless, certain ways lead to the solution more straightforwardly and rapidly. We have found the following general guidelines work well for MK2 as well as other protein targets 


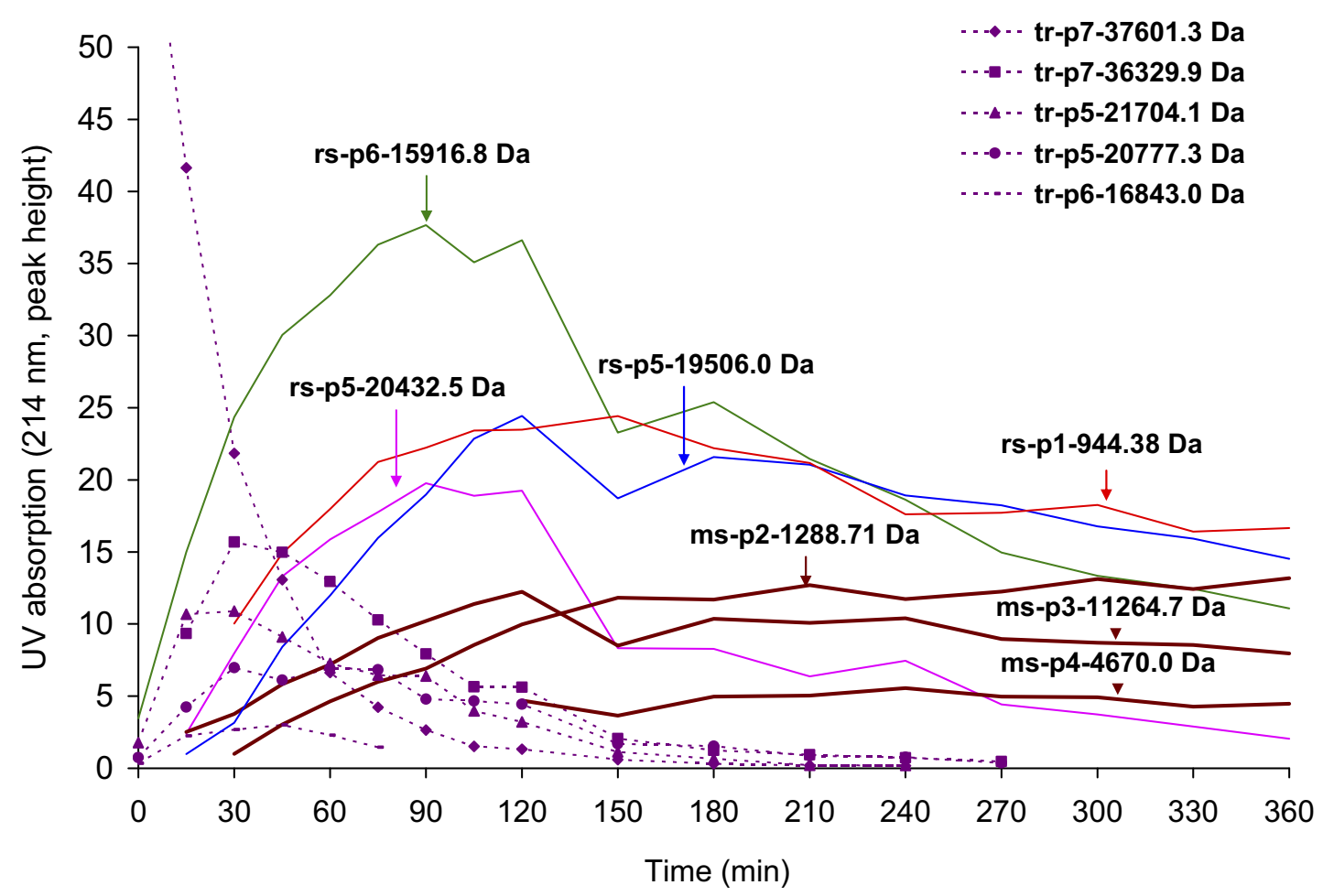

Figure 2. Time dependence of major proteolytic fragments of MK2 observed by LC/MS. UV (214 $\mathrm{nm})$ peak height is used directly for quantification if only one mass was detected in the peak; if multiple masses were detected, the relative content of each component was approximated from the mass spectral intensity, assuming the fragments have similar ionization potentials. Fragments are labeled as most stable (ms), relatively stable (rs), or transient (tr), along with the LC peak they eluted in, and the observed mass.

in our lab: (1) identify larger fragments first because they usually have fewer potential sequences and may in fact be unambiguously identified from the observed mass value alone; (2) during the initial cleavage period, look for complementary fragments whose masses sum to that of the intact full-length protein (after subtracting the mass of water due to hydrolysis of the peptide bond); (3) start from the beginning of the time course, analyze the late stage next, and, finally, work toward the intermediate stages; and (4) fragments that elute from the same chromatographic peak are structurally similar and may contain the same amino or carboxyl terminus.

List fragment masses against potential sequences. As mentioned previously, the list is a set of sequences whose calculated masses are within a small mass window (experimental error) of the detected mass. Two factors are considered when setting the width of the mass window. The mass window should be large enough to include the true sequence, but not be too large to introduce false positives. It is therefore obvious that the higher the accuracy of the mass spectrometer used, the easier it is to narrow down the true sequence. Since our LCQ-DUO mass spectrometer can routinely achieve a mass accuracy of better than $\pm 2 \mathrm{Da}$ after recalibration in the mass range up to $40 \mathrm{kD}$, a mass window of $\pm 3 \mathrm{Da}$ was chosen to select potential sequences for most measured masses that required deconvoluting multiply charged ions. For smaller fragments $(<2000 \mathrm{Da})$ whose masses were measured directly by monoisotopic mass with higher precision $(<0.1 \mathrm{Da})$, a mass window of \pm 0.2 Da was chosen. Thus, average masses and monoisotopic masses were used for large ( $>2000 \mathrm{Da}$ ) and small $(<2000 \mathrm{Da})$ fragments, respectively. For each of the detected fragments shown in Tables 2-4, all possible sequences and their masses were calculated by a custom-written Perl script.

Identify large fragments and complementary fragments during initial cleavage. The initial cleavage period produces key information that greatly facilitates identification of the various intermediates and final limited proteolysis fragments of ultimate interest. For example, during the initial cleavage period, it is possible to generate large fragments that can be readily identified because there may be only a single sequence region whose mass matches with it. This is in fact the case for tr-p7-36,329.9 Da (Table 3), which was identified as (11-325), because it is the only possible sequence within the selection window of $\pm 3 \mathrm{Da}$.

Another key piece of information obtainable during the initial cleavage events is the production of pairs of masses, whose values sum to the total mass of either the 
Table 2. Detected complementary fragments of MK2 and their potential sequences. ${ }^{\mathrm{a}}$

\begin{tabular}{|c|c|c|c|c|c|c|c|}
\hline \multicolumn{4}{|c|}{ (a) Complementary fragments } & \multicolumn{4}{|c|}{ (b) Complementary fragments } \\
\hline \multicolumn{2}{|c|}{ rs-p6-15916.8 $\pm 0.5 \mathrm{Da}$} & \multicolumn{2}{|c|}{$r-p 5-21704.1 \pm 1.1 \mathrm{Da}$} & \multicolumn{2}{|c|}{$\operatorname{tr}-\mathrm{p} 6-16843.0 \pm 1.2 \mathrm{Da}$} & \multicolumn{2}{|c|}{$\operatorname{tr}-\mathrm{p} 5-20777.3 \pm 1.1 \mathrm{Da}$} \\
\hline $\begin{array}{l}\text { Potential } \\
\text { sequence }\end{array}$ & $\begin{array}{c}\text { Calculated } \\
\text { mass }\end{array}$ & $\begin{array}{l}\text { Potential } \\
\text { sequence }\end{array}$ & $\begin{array}{c}\text { Calculated } \\
\text { mass }\end{array}$ & $\begin{array}{l}\text { Potential } \\
\text { Sequence }\end{array}$ & $\begin{array}{c}\text { Calculated } \\
\text { mass }\end{array}$ & $\begin{array}{l}\text { Potential } \\
\text { sequence }\end{array}$ & $\begin{array}{c}\text { Calculated } \\
\text { mass }\end{array}$ \\
\hline$(1-138)$ & 15915.51 & $(1-189)$ & 21703.06 & $(1-146)$ & 16844.56 & $(1-181)$ & 20776.02 \\
\hline$(6-144)$ & 15919.48 & $(3-191)$ & 21706.04 & $(8-154)$ & 16846.55 & $(7-188)$ & 20780.00 \\
\hline$(9-147)$ & 15915.45 & (18-207) & 21702.93 & (54-200) & 16842.31 & $(12-193)$ & 20774.98 \\
\hline$(10-148)$ & 15915.45 & $(20-208)$ & 21703.96 & $(121-267)$ & 16840.39 & $(42-221)$ & 20775.00 \\
\hline (11-149) & 15915.45 & $(31-220)$ & 21705.16 & $(122-268)$ & 16839.44 & $(46-225)$ & 20776.97 \\
\hline$(32-169)$ & 15915.45 & $(39-226)$ & 21707.09 & $(145-289)$ & 16844.46 & $(70-251)$ & 20780.87 \\
\hline$(33-170)$ & 15915.45 & $(52-241)$ & 21707.11 & $(161-306)$ & 16840.46 & $(71-252)$ & 20780.87 \\
\hline (82-221) & 15915.24 & (63-251) & 21700.91 & $(165-309)$ & 16842.44 & $(79-260)$ & 20775.78 \\
\hline (83-222) & 15913.23 & $(92-280)$ & 21701.00 & (182-325) & 16843.50 & $(103-283)$ & 20780.72 \\
\hline (93-233) & 15919.28 & (108-295) & 21706.91 & & & (112-291) & 20777.82 \\
\hline (99-239) & 15920.18 & $(115-303)$ & 21707.98 & & & (119-298) & 20779.97 \\
\hline$(104-244)$ & 15914.21 & $(125-311)$ & 21704.03 & & & $(126-305)$ & 20773.06 \\
\hline$(111-250)$ & 15915.19 & $(127-313)$ & 21702.06 & & & $(128-307)$ & 20774.05 \\
\hline$(128-266)$ & 15912.38 & $(139-325)$ & 21704.01 & & & $(145-323)$ & 20780.91 \\
\hline$(159-296)$ & 15914.43 & & & & & $(146-324)$ & 20774.96 \\
\hline$(163-300)$ & 15914.42 & & & & & $(147-325)$ & 20774.96 \\
\hline$(176-311)$ & 15919.36 & & & & & & \\
\hline$(190-325)$ & 15916.46 & & & & & & \\
\hline \multicolumn{2}{|c|}{$\Delta$ mass $=0.3 \mathrm{Da}$} & \multicolumn{2}{|c|}{$\Delta$ mass $=1.0 \mathrm{Da}$} & \multicolumn{2}{|c|}{$\Delta$ mass $=-0.5 \mathrm{Da}$} & \multicolumn{2}{|c|}{$\Delta$ mass $=1.3 \mathrm{Da}$} \\
\hline
\end{tabular}

${ }^{a}$ Fragment nomenclature is described in the legend to Figure 2. Sequence regions are indicated by the sequence number of the amino and carboxyl terminal residues separated by a hyphen. For each detected fragment mass, a mass window of at least \pm 3 Da was used to select potential sequences. Sequences not satisfying a complementary relationship (sequences if fused together would form the original molecule) are eliminated and shown in gray. The final determined sequences are shown in bold. Each $\Delta$ mass is the difference between detected mass and the calculated mass of its determined sequence.

Table 3. Four detected fragments and their potential sequences. ${ }^{\mathrm{a}}$

\begin{tabular}{|c|c|c|c|c|c|c|c|}
\hline \multicolumn{2}{|c|}{ tr-p7-36329.9 $\pm 1.1 \mathrm{Da}$} & \multicolumn{2}{|c|}{ rs-p5-19506.0 $\pm 0.9 \mathrm{Da}$} & \multicolumn{2}{|c|}{ rs-p5-20432.0 $\pm 0.7 \mathrm{Da}$} & \multicolumn{2}{|c|}{ ms-p3-11264.7 $\pm 0.5 \mathrm{Da}$} \\
\hline $\begin{array}{l}\text { Potential } \\
\text { sequence }\end{array}$ & $\begin{array}{c}\text { Calculated } \\
\text { mass }\end{array}$ & $\begin{array}{l}\text { Potential } \\
\text { sequence }\end{array}$ & $\begin{array}{c}\text { Calculated } \\
\text { mass }\end{array}$ & $\begin{array}{l}\text { Potential } \\
\text { Sequence }\end{array}$ & $\begin{array}{c}\text { Calculated } \\
\text { mass }\end{array}$ & $\begin{array}{l}\text { Potential } \\
\text { sequence }\end{array}$ & $\begin{array}{c}\text { Calculated } \\
\text { mass }\end{array}$ \\
\hline \multirow[t]{18}{*}{$(11-325)$} & 36330.00 & $(1-169)$ & 19502.65 & $(8-186)$ & 20434.61 & $(3-99)$ & 11267.34 \\
\hline & & $(6-175)$ & 19504.61 & (11-189) & 20431.56 & $(2-98)$ & 11264.28 \\
\hline & & (11-181) & 19504.52 & $(21-199)$ & 20433.48 & $(4-100)$ & 11268.32 \\
\hline & & $(53-222)$ & 19508.53 & (87-265) & 20429.46 & (79-177) & 11265.88 \\
\hline & & $(58-227)$ & 19503.37 & $(89-267)$ & 20428.52 & $(105-203)$ & 11266.66 \\
\hline & & $(64-234)$ & 19508.37 & $(93-271)$ & 20436.53 & $(118-216)$ & 11263.88 \\
\hline & & (83-253) & 19507.31 & $(95-273)$ & 20434.46 & $(126-224)$ & 11263.04 \\
\hline & & $(101-271)$ & 19506.30 & (119-295) & 20432.55 & $(130-228)$ & 11262.89 \\
\hline & & $(102-272)$ & 19504.27 & $(123-300)$ & 20434.57 & $(140-239)$ & 11263.00 \\
\hline & & $(105-274)$ & 19503.29 & $(150-325)$ & 20432.56 & $(151-249)$ & 11267.99 \\
\hline & & $(109-278)$ & 19503.35 & & & $(156-254)$ & 11263.91 \\
\hline & & $(115-284)$ & 19503.36 & & & $(158-256)$ & 11261.94 \\
\hline & & $(126-294)$ & 19510.59 & & & $(159-257)$ & 11262.88 \\
\hline & & $(145-311)$ & 19504.48 & & & (194-289) & 11262.12 \\
\hline & & $(151-317)$ & 19503.46 & & & $(196-291)$ & 11263.15 \\
\hline & & & & & & $(217-311)$ & 11263.03 \\
\hline & & & & & & $(218-312)$ & 11264.96 \\
\hline & & & & & & $(231-325)$ & 11264.02 \\
\hline \multicolumn{2}{|c|}{$\Delta$ mass $=-0.1 \mathrm{Da}$} & \multicolumn{2}{|c|}{$\Delta$ mass $=1.5 \mathrm{Da}$} & \multicolumn{2}{|c|}{$\Delta$ mass $=0.9 \mathrm{Da}$} & \multicolumn{2}{|c|}{$\Delta$ mass $=0.7 \mathrm{Da}$} \\
\hline
\end{tabular}

aSequences eliminated in the first step are shown in gray; the final determined sequences are shown in bold. Each $\Delta$ mass is the difference between detected mass and the calculated mass of its determined sequence. For the fragment tr-p7-36329.9 Da, there is only one potential sequence within the $\pm 3 \mathrm{Da}$ window. 
intact protein or a major identifiable fragment thereof. For example, identification of tr-p7-36,329.9 Da as (11325 ) is further supported by the observation of the expected complementary small fragment mass of msp2-1288.71 Da. That is, the sum of the two fragment masses (minus the mass of a water molecule) is equal to the full-length mass of MK2 (37,601.5 Da). This detected smaller mass also differed by only 0.07 Da from the calculated mass $(1288.64 \mathrm{Da})$ of $(1-10)$. It was thus concluded with a high degree of certainty that these two fragments resulted from cleavage of MK2 at site $(10,11)$ (here we annotate cleavage sites by the two residues at the amino and carboxyl sides of the site, separated by a comma).

The concept of mass additivity and sequence complementarity also helped with some of the other fragments observed early in the limited proteolysis reaction. In the first time point ( $2 \mathrm{~min})$, four fragment masses were detected in HPLC peaks 5 and $\mathbf{6}$ (Table 1). It was straightforward to infer that rs-p6-15,916.8 Da and tr-p5-21,704.1 Da are complementary fragments for two reasons. First, the sum of these masses (minus the 18 Da mass for proteolytic addition of a water molecule) equals $37,602.9 \mathrm{Da}$ and is within error equal to the calculated mass of intact MK2 $(37,601.5 \mathrm{Da})$. Second, complementary (mutually exclusive) sequence regions of MK2 have mass values that match the masses of these fragments (Table 2a). Likewise, similar relationships exist for tr-p6-16,843.3 Da and tr-p5-20,777.3 Da. With the complementary relationships identified for these two pairs of fragments, all potential sequences except those containing the original $\mathrm{N}$ - or C-terminus were eliminated, as shown in gray in Table 2.

Interestingly, despite having eliminated the majority of potential sequences, there still remain two possibilities for the identities of the complementary fragments rs-p6-15,916.8 Da and tr-p5-21,704.1 Da. That is, two sets of complementary sequence regions from MK2 sum to the mass of the intact MK2 molecule (from Table 2a): (1-138) and (139-325) as well as (1-189) and (190-325). Similarly, there still remain two combinations for the fragments tr-p6-16,843.3 Da and tr-p5-20,777.3 Da: both (1-146) and (147-325) as well as (1-181) and (182-325) sum to, within error, the mass of intact MK2 as shown in Table $2 b$.

With the prior identification of the cleavage site (10, 11 ), the orientation of the above two pairs of complementary fragments can easily be resolved. For the stable fragment rs-p6-15,916.8 Da, its identity had been narrowed down to either (1-138) or (190-325). If its identity were the former, containing the cleavage site (10, $11)$, an equally or more stable fragment (11-138), with a calculated mass of $14,645.0 \mathrm{Da}$, would have been generated but was not detected at any time point along the entire reaction. This supports assignment of rs-p6$15,916.8 \mathrm{Da}$ to $(190-325)$. This in turn predicts that its complementary fragment, tr-p5-21,704.1 Da, now tentatively assigned as (1-189), would also lead to a more stable fragment (11-189) resulting from cleavage at (10,
11), with a calculated mass of 20,431.6 Da. A fragment (rs-p5-20,432.5) with this mass is indeed observed as a relatively stable fragment co-eluting in peak 5 at later time points. Thus fragment rs-p6-15,916.8 $\mathrm{Da}$ is assigned as (190-325), tr-p5-21,704.1 $\mathrm{Da}$ as $(1,189)$, and rs-p5-20,432.5 as (11-189).

Identification of the other pair of complementary fragments (tr-p6-16,843.3 Da and tr-p5-20,777.3 Da) was facilitated by their LC elution behavior. Although the LC elution profiles were not as rigorous in identifying the fragments as the mass spectrometry data were, they did provide valuable insight to guide data interpretation. As an example, the fragments tr-p6-16,843.3 Da and rs-p6-15,916.8 Da have similar masses and both appeared in peak 6 in HPLC separation; likewise, fragments tr-p5-21,704.1 Da and tr-p5-20,777.3 Da have similar masses and both appeared in peak 5 in HPLC separation. This indicates that they were two groups of fragments having similar physical properties and strongly suggests they could consist of very similar sequence regions. Since rs-p6-15,916.8 Da was determined to be (190-325) containing the original C-terminus, it was most likely that the unidentified fragment tr-p6-16,843.3 Da could also be a C-terminal fragment (182-325). A similar argument also exists for fragments tr-p5-21,704.1 Da and tr-p5-20,777.3 Da. Since tr-p521,704.1 Da has been identified as (1-189) containing the original N-terminus, the fragment tr-p5-20,777.3 Da is most likely the $\mathrm{N}$-terminus containing (1-181). These assignments indicate that MK2 has two more cleavage sites at $(181,182)$ and $(189,190)$, in addition to the already identified $(10,11)$. Importantly, additional support for these assignments, and all assignments, comes from self-consistency in analyzing the global dataset from the early, mid, and late stages of limited proteolysis.

Identify fragments from combinations of cleavage sites. At this point, a total of three cleavage sites at $(10,11),(181$, $182)$, and $(189,190)$ have been identified as shown in Figure 3. One way to identify more fragments is to search for all possible fragments that result from various combinations of already identified cleavage sites and see whether the calculated masses match with detected masses. For example, knowing the presence of three cleavage sites at $(10,11),(181,182)$, and $(189,190)$, the calculated masses for the two sequences, (11-181) and (182-189), are 19,504.5 and $944.40 \mathrm{Da}$, respectively. By examining the remaining unidentified masses (Table $1)$, one can assign the two fragments rs-p5-19,506.0 Da and rs-p1-944.38 Da to these two sequences. By this approach, the only remaining unidentified fragment from peak 5 can be identified. However, for the purpose of illustrating additional constraints that exist in the current dataset, the subsequent discussion demonstrates other means by which the two fragments, rs-p519,506.0 Da and rs-p5-20,432.5 Da, are identified. 
MK2 (325 amino acids, calculated mass $=37601.5 \mathrm{Da})$

181182

189190

230231 325

\subsection{Da}

\begin{tabular}{|l|l|l|r}
$19504.5 \mathrm{Da}$ & $944.4 \mathrm{Da}$ & $4670.5 \mathrm{Da}$ & $11264.0 \mathrm{Da}$ \\
\hline
\end{tabular}

Figure 3. Schematic of identified cleavage sites on MK2 cleaved by subtilisin and resulting fragments. Shown on each fragment is the calculated mass of the assigned sequence. The shade of each bar represents the fragment's relative stability, with darker ones indicating greater stability. The fragments with calculated masses of $1288.6,944.4,4670.5$, and 11,264.0 Da are the most stable, followed by relatively stable ones at 19,504.5, 20,431.6, and 15,916.5 Da; the rest are transient fragments.

Identify fragments by subfragment relationship. Shown in Figure 4 are mass spectra of peak 5 at three selected time points representing the early, middle, and late stages of proteolysis. We have found that proteolytic

fragments that co-elute by LC often contain a common sequence region and similar length, thus giving rise to their similar chromatographic behavior. It is evident that tr-p5-21,704.1 $\mathrm{Da}$ and tr-p5-20,777.3 Da were

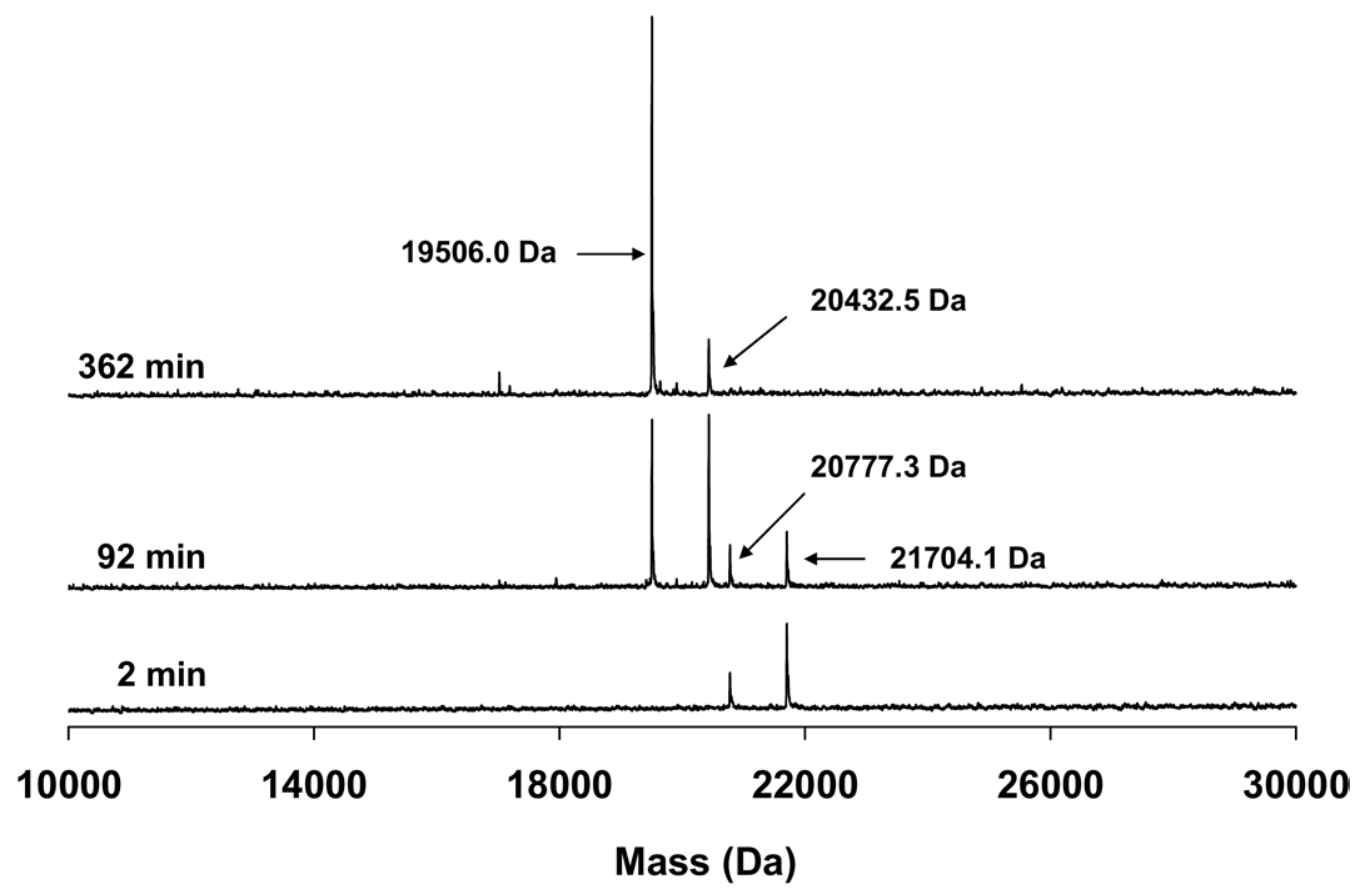

Figure 4. Mass spectra of peak 5 at selected time points representing the early, middle, and late stages of proteolysis reaction. These spectra were obtained by deconvoluting multiply charged fragment ions to transfer from the raw $\mathrm{m} / \mathrm{z}$ spectrum to the zero charge mass domain. It is evident that initially formed fragments at 21,704.1 and 20,777.3 Da were continuously cleaved to form the fragments at 20,432.5 and 19,506.0 Da. Eventually, the fragment at 19,506.0 Da appeared to be the most stable among them. 
Table 4. Detected small fragments in peaks 1,2 , and 4 , and their potential sequences. ${ }^{\text {a }}$

\begin{tabular}{|c|c|c|c|c|c|}
\hline \multicolumn{2}{|c|}{ rs-p1-944.38 $\pm 0.03 \mathrm{Da}$} & \multicolumn{2}{|c|}{ ms-p2-1288.71 $\pm 0.03 \mathrm{Da}$} & \multicolumn{2}{|c|}{ ms-p4-4570.0 $\pm 0.0 \mathrm{Da}$} \\
\hline $\begin{array}{l}\text { Potential } \\
\text { sequence }\end{array}$ & $\begin{array}{c}\text { Calculated } \\
\text { mass }\end{array}$ & $\begin{array}{l}\text { Potential } \\
\text { sequence }\end{array}$ & $\begin{array}{c}\text { Calculated } \\
\text { mass }\end{array}$ & $\begin{array}{l}\text { Potential } \\
\text { Sequence }\end{array}$ & $\begin{array}{c}\text { Calculated } \\
\text { mass }\end{array}$ \\
\hline \multirow[t]{13}{*}{ (182-189) } & 944.40 & $(1-10)$ & 1288.64 & $(62-100)$ & 4667.49 \\
\hline & & (139-149) & 1288.70 & $(65-104)$ & 4672.47 \\
\hline & & $(172-183)$ & 1288.63 & $(78-118)$ & 4672.41 \\
\hline & & $(232-242)$ & 1288.71 & $(146-186)$ & 4671.30 \\
\hline & & & & (158-199) & 4671.30 \\
\hline & & & & $(159-200)$ & 4671.30 \\
\hline & & & & $(190-230)$ & 4670.45 \\
\hline & & & & (195-236) & 4667.50 \\
\hline & & & & $(200-240)$ & 4670.55 \\
\hline & & & & $(202-242)$ & 4666.58 \\
\hline & & & & $(221-260)$ & 4673.30 \\
\hline & & & & $(235-273)$ & 4667.39 \\
\hline & & & & (258-296) & 4669.55 \\
\hline \multicolumn{2}{|c|}{$\Delta$ mass $=-0.02 \mathrm{Da}$} & \multicolumn{2}{|c|}{$\Delta$ mass $=0.07 \mathrm{Da}$} & \multicolumn{2}{|c|}{$\Delta$ mass $=-0.5 \mathrm{Da}$} \\
\hline
\end{tabular}

${ }^{a}$ For the two smallest fragments rs-p1-944.38 Da and ms-p2-1288.71 Da, which were measured by monoisotopic mass, a mass window of $\pm 0.2 \mathrm{Da}$ was used to select potential sequences. The final determined sequences are shown in bold. Each $\Delta$ mass is the difference between detected mass and the calculated mass of its determined sequence. For the fragment rs-p1-944.38 $\pm 0.03 \mathrm{Da}$, there is only one potential sequence within the \pm 0.2 Da window.

formed first; they gradually collapsed down to rs-p520,432.5 Da and rs-p5-19,506.0 Da, and eventually rsp5-19,506.0 Da appeared to be the most stable fragment among them. This time course suggests the two fragments, rs-p5-19,506.0 Da and rs-p5-20,432.5 Da, must be subfragments of tr-p5-21,704.1 Da, the largest one within the group. In other words, rs-p5-19,506.0 Da and rs-p5-20,432.5 Da should not have a terminus longer than that of tr-p5-21,704.1 Da, whose identity has already been determined to be (1-189). Therefore, potential sequences for rs-p5-19,506.0 Da and rs-p5-20,432.5 Da that do not satisfy this relationship were eliminated and are shown in gray in Table 3.

At this point, there are still three remaining possible sequences for rs-p5-19,506.0 Da: (1-169), (6-175), and (11-181). Since rs-p5-19,506.0 Da was one of the more stable fragments except those most stable ones (Figure $2)$, it was unlikely to contain the susceptible cleavage site $(10,11)$. Furthermore, for $(1-169)$ or $(6-175)$ to be true identities for any fragments, the cleavage sites (169, $170)$ or $(5,6)$ and $(175,176)$ would have to exist, respectively. However, there was no evidence supporting either hypothesis. Therefore, the only reasonable assignment for rs-p5-19,506.0 Da is (11-181), as shown in bold in Table 3. For rs-p5-20,432.5 Da, the two remaining possible sequences are (8-186) and (11-189). Between them, only (11-189) appeared to be the real sequence. This is because additional cleavage sites at $(7$, 8 ) and $(186,187)$ would have to exist to make $(8-186)$ a reasonable choice. On the other hand, since $(10,11)$ and $(189,190)$ have already been identified as cleavage sites, cleaving at $(10,11)$ and $(189,190)$ would readily generate (11-189). Therefore, (11-189) is assigned to rs-p520,432.5 Da.
Identify the most stable fragments remaining late in the time course. To this point, the only two observed fragments yet to be identified are ms-p3-11,264.7 Da and ms-p4$4670.0 \mathrm{Da}$. Since they were two of the most stable fragments (Figure 2), they were unlikely to contain any of the cleavage sites identified so far. Otherwise, they would have been cleaved during the time course. Therefore, they could only evolve from either (11-181) at $19,506.0 \mathrm{Da}$ or (190-325) at $15,916.806 .5 \mathrm{Da}$, two of the fragments that do not contain previously identified cleavage sites as shown in Figure 3. Consequently, potential sequences for ms-p3-11,264.7 Da that are not covered by (11-181) or (190-325) are ruled out and shown in gray.

By examining the remaining sequences for ms-p311,264.7 Da in Table 3, if it evolved from (11-181), the only choice would be (79-177). If it evolved from (190-325), several sequences seem to be possible (the bottom 5 on the list in Table 3). However, since it was formed by additional cleaving of a relatively stable fragment, very limited additional cleaving is expected during its formation. Therefore, sequence (231-325), which could come from a single cleavage on (190-325), appeared to be the most reasonable choice. Any other sequences would require two more cleaving events to be generated, and there was no evidence indicating the existence of these extra cleavage sites. Furthermore, strong additional evidence supporting this assignment is that its complementary fragment (190-230), with a calculated mass of $4670.45 \mathrm{Da}$ and presumably cleaved from (190-325) when (231-325) was generated, was in fact found in peak 4 at $4670.0 \mathrm{Da}$. With the identification of the two most stable fragments, ms-p3-11,264.7 Da and ms-p4-4670.0 Da, a fourth cleavage site at $(230,231)$ 


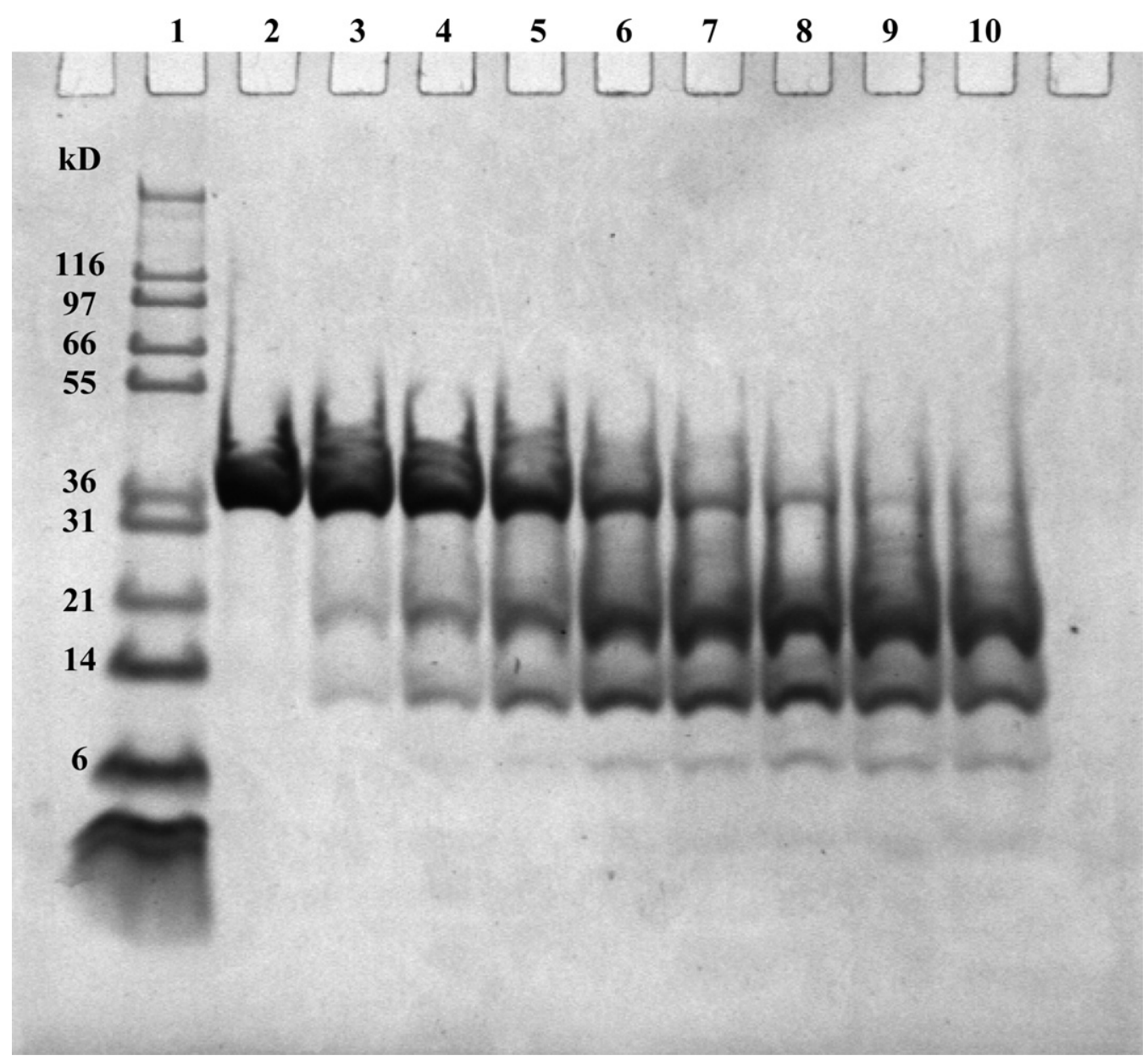

Figure 5. Time-dependent limited proteolysis of MK2 visualized by SDS-PAGE. MK2 at $1 \mathrm{mg} / \mathrm{mL}$ was mixed with subtilisin with an $\mathrm{E} / \mathrm{S}$ ratio of $1 / 20 \mathrm{~K}$ at time zero and incubated at $25^{\circ} \mathrm{C}$. A total of $10 \mu \mathrm{L}$ of reaction mixture from nine time points was loaded as follows: Mark $12^{\mathrm{TM}}$ protein standards (lane 1), 2 min (lane 2), $17 \mathrm{~min}$ (lane 3), $32 \mathrm{~min}$ (lane 4), $1 \mathrm{~h}$ (lane 5), $2 \mathrm{~h}$ (lane 6), $3 \mathrm{~h}$ (lane 7), $4 \mathrm{~h}$ (lane 8), $5 \mathrm{~h}$ (lane 9), and $6 \mathrm{~h}$ (lane 10). Overloading was done to make the weaker bands more visible. Intact MK2 migrated with an apparent molecular weight of $37 \mathrm{kDa}$. When MK2 molecules were cleaved by subtilisin, three groups of fragments in the approximate ranges of 18-30, 12-16, and $7 \mathrm{kDa}$ were formed, respectively.

was also identified. The complete cleavage map of MK2 depicting all identified cleavage sites and resulting fragments is summarized in Figure 3.

After all proteolysis products are identified by the current method, we compared the difference between each detected mass and the calculated mass of its determined identity as shown in the last rows in Tables 2,3 , and 4 . The differences were from $-0.2 \mathrm{Da}$ to +1.5 $\mathrm{Da}$. This distribution was well within the mass window $( \pm 3 \mathrm{Da})$ used in selecting potential sequences. This indicated that the mass window used in selecting potential sequences was large enough. With all of the major proteolysis reaction products identified, a dynamic picture of the entire proteolysis process was assembled by combining the information shown in Figures 2 and 3. It was evident that none of the fragments containing $(10,11)$ or $(189,190)$ was stable. In contrast, the relatively stable fragments, (11-189) and (190-325), contained cleavage sites $(181,182)$ and $(230,231)$, respectively. This indicated the two cleavage sites at $(10,11)$ and $(189,190)$ were more susceptible to proteolysis than the two at $(181,182)$ and $(230,231)$.

\section{Comparison to SDS-PAGE and N-Terminal Sequencing Data}

To compare the present method to the more traditional approach of relying on SDS-PAGE and N-terminal sequencing, as well as to help validate the present method, we conducted SDS-PAGE and N-terminal sequencing analyses under conditions very similar to those of the time-resolved LC/MS study. Figure 5 shows a time course for the limited proteolysis reaction of MK2. There are clearly multiple bands, but it is not clear how many could be overlapping by SDS-PAGE analysis. Traditionally, $\mathrm{N}$-terminal sequencing is done 


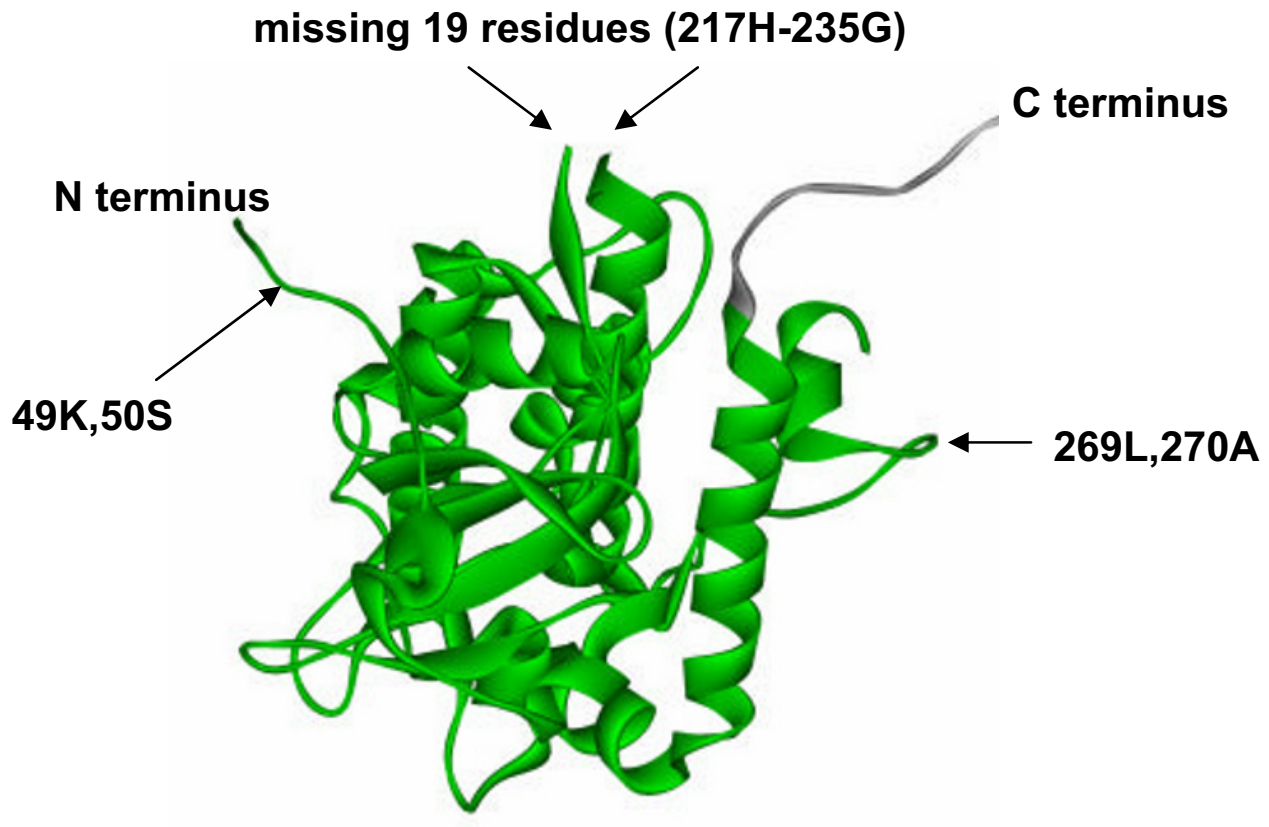

Figure 6. Crystal structure of MK2 (1KWP.pdb) [32]. The four cleavage sites identified by limited proteolysis, (49Lys, 50Ser), (220Leu, 221Thr), (228Tyr, 229Tyr), and (269Leu, 270Ala), are all exposed and apparently flexible (numbering is from 1KWP.pdb and shifted by 39 from that used in the present study). Two of the identified cleavage sites, (220Leu, 221Thr) and (228Tyr, 229Tyr), are on the activation loop and fall in the region of 19 unresolved residues $(217 \mathrm{H}-235 \mathrm{G})$ in crystal structure, presumably due to the high degree of flexibility of this region. The C-terminal 21 residues 365-385, which are absent in the construct of present study as shown in gray, extend into solution and away from the core structure. The limited proteolysis and X-ray crystal structure data are in good agreement based on the concept that limited proteolysis cleaves preferentially at exposed and flexible regions.

on bands that appear to reflect stable domains. We did $\mathrm{N}$-terminal sequencing on the major bands of the 1-h time point sample that migrated with apparent molecular weight (estimated using Mark12 ${ }^{\mathrm{TM}}$ protein standard) of 19, 13, and $7 \mathrm{kD}$, respectively. Their first 5 amino acids at the N-terminus were (11-15), (190-194), and (190-194), respectively. These bands correspond to rs-p5-20,432.5 $\mathrm{Da}$ and/or rs-p5-19,506.0 Da, rs-p615,916.8 Da, and ms-p4-4670.0 Da, respectively. These results confirmed the cleavage sites previously identified by the LC/MS only approach. Since the molecular weight measurement by SDS-PAGE lacks enough precision, even when N-terminal sequencing data are available, it can still be difficult to determine the exact C-termini of the fragments. Mass spectrometry analysis of the bands by the proteomics approach would obviously greatly facilitate identification of the fragments, but in most cases would likely require the $\mathrm{N}$-terminal sequencing data for unambiguous determination. Furthermore, it is extremely tedious to obtain dynamic information of proteolysis as shown in Figure 2 by the proteomics approach. As a contrast, the time-resolved LC/MS only approach provides significantly more information about the proteolysis process and it is also less time-consuming.

\section{Comparison to Crystal Structure Data}

We mapped the cleavage sites identified by the current method onto the known crystal structure of MK2, shown in Figure 6 [32], to determine whether their locations correspond to flexible or surface-exposed regions. The protein crystal structure was obtained with a construct of MK2 consisting of amino acids (1-400), which relative to the construct used in this study contained an extra 39 amino acids at the N-terminus and an extra 36 amino acids at the C-terminus. Assignable electron density was observed for the region spanning (46-385), covering residues (7-325) in the construct used in this study, with an extra 36 amino acids at the C-terminus and two gaps at (217-235) and (282$283)$, respectively. The 21 residues at the C-terminus (365-385), which were absent in the construct of the present study, extended into solution and away from the core structure as shown in gray, apparently causing no distortion to the core structure. The identified cleavage site $(10,11)$ corresponds to (49Lys, 50Ser) in crystal structure, which is located on a partially unfolded extended strand at the $\mathrm{N}$-terminus. The two cleavage sites $(180,181)$ and $(189,190)$ correspond to $(220 \mathrm{Leu}$, $221 \mathrm{Thr})$ and (228Tyr, 229Tyr), respectively, in the crys- 
tal structure. They fell within a missing gap of 19 amino acids in the activation loop for which there was no electron density visible in crystal structure. The missing gap was presumably caused by a high degree of flexibility, such that not enough electron density was registered for any single conformation. The cleavage site $(230,231)$ corresponds to $(269 \mathrm{Leu}, 270 \mathrm{ALa})$ in the crystal structure, which was located on an exposed linker region between two helices. The four observed cleavage sites all appeared to be exposed, and at least two of them were in regions of high flexibility. Overall, three areas of the MK2 molecule were sensitive to proteolysis, and only four sites were preferentially cleaved. It is also worth pointing out that stable fragments generated by limited proteolysis are based on structural stability; they may not correspond to fully functional domains. This is because some functional domains have flexible loops in the middle that are susceptible to proteolysis, as is in the case of MK2 where its activation loop was among the first to be cleaved.

\section{Conclusions}

We have described a novel, time-resolved LC/MS approach for identifying stable domains and flexible regions on the catalytic domain of MK2 by limited proteolysis. This method eliminated the need for conducting additional experiments with other technologies such as SDS-PAGE, N-terminal sequencing, and peptide mapping. Importantly, it took less time and was more informative than traditional approaches. The time-resolved approach not only enabled identification of the most stable fragments, but also provided insight into the dynamic nature of the limited proteolysis reaction at early and intermediate stages of proteolysis. Since it does not require specific proteases, it is a more universal method that can be used in any limited proteolysis studies where proteins are cleaved based on structural flexibility and surface exposure. Currently, with a regular ion-trap type of mass spectrometer, a target protein with a mass up to $50 \mathrm{kDa}$ can be readily analyzed. For the current method to be applied successfully, it is essential that the 6-h time course captures all critical steps of proteolysis-that is, from the initial cleaving to generate complementary fragments, through different intermediate states-until all stable fragments are formed. This will usually allow enough constraints or relationships governing proteolytic fragments to be identified. Similar to solving equations of multiple unknowns, as long as enough constraints can be found, all proteolytic fragments can be determined robustly. That is, because all fragments are intrinsically linked by specific relationships, incorrect assignment of some fragments will invariably cause others to be without a valid solution. This greatly reduces the likelihood that assignment errors early in the process would be propagated throughout the process. We have also applied this method successfully to more than 10 unrelated recombinant proteins so far in our laboratory (unpub- lished results). We have found that if a clear mass spectrum was obtained on an intact target protein, its proteolytic fragments were also measured reliably and the current method was applicable. Because this method identifies flexible and stable regions of proteins, it is useful in the design of protein constructs for X-ray crystallography studies [29], as well as for studying proteins with unknown structures. It is also conceivable that when a high-resolution mass spectrometer such as Fourier transform mass spectrometer (FTMS) is used, this method can be applied to much larger proteins, since the corresponding mass window for selecting potential subsequences can be significantly reduced for FTMS. In addition, FTMS can perform sequencing "on the fly" on protein fragments, thus achieving rapid analysis with an even higher confidence level [33, 34]. Given the relatively simple mathematical mass constraints of the proteolytic products generated from a target protein of known sequence, it should be possible to globally analyze by automated computational methods.

\section{Acknowledgments}

We thank Matt Pokross and John Newitt from the Department of Gene Expression and Protein Biochemistry, Bristol-Myers Squibb Co., for their invaluable suggestions during preparation of this manuscript.

\section{References}

1. Hubbard, S. J. The Structural Aspects of Limited Proteolysis of Native Proteins. Biochim. Biophys. Acta 1998, 1382, 191-206.

2. Fontana, A.; de Laureto, P. P.; Spolaore, B.; Frare, E.; Picotti, P. Zambonin, M. Probing Protein Structure by Limited Proteolysis. Acta Biochim. Polonica 2004, 51, 299-321.

3. Zhao, Y.; Muir, T. W.; Kent, S. B. H.; Tischer, E.; Scardina, J. M.; Chait, B. T. Mapping Protein-Protein Interactions by Affinity-Directed Mass Spectrometry. Proc. Natl. Acad. Sci. U.S.A. 1996, 93, 4020-4024.

4. Gervasoni, P.; Staudenmann, W.; James, P.; Plückthun, A. Identification of the Binding Surface on $\beta$-Lactamase for GroEL by Limited Proteolysis and MALDI-Mass Spectrometry. Biochemistry 1998, 37, 11660-11669.

5. Kriwacki, R. W.; Wu, J.; Siuzdak, G.; Wright, P. E. Probing Protein/ Protein Interactions with Mass Spectrometry and Isotopic Labeling: Analysis of the p21/Cdk2 Complex. J. Am. Chem. Soc. 1996, 118, 5320-5321.

6. Seielstad, D. A.; Carlson, K. E.; Kushner, P. J.; Greene, G. L.; Katzenellenbogen, J. A. Analysis of the Structural Core of the Human Estrogen Receptor Ligand Binding Domain by Selective Proteolysis/Mass Spectrometric Analysis. Biochemistry 1995, 34, 12605-12615.

7. Teffera, Y.; Bakhtiar, R. Confirmation of the Binding Site of CyclophilinCyclosporin Non-covalent Complex Using Limited Proteolysis and Liquid Chromatography-Quadrupole-Time-of-Flight Mass Spectrometer. J. Liquid Chromatogr. Relat. Technol. 2005, 28, 1497-1508.

8. Kriwacki, R. W.; Wu, J.; Tennant, L.; Wright, P. E.; Siuzdak, G. Proteolysis, Matrix-Assisted Laser Desorption/Ionization Mass Spectrometry, High-Performance Liquid Chromatography and Size-Exclusion Chromatography of $21^{\text {Wafi/Cip1/Sdi1 }}$. J. Chromatogr. A 1997, 777 , 23-30.

9. Aghajanian, S.; Hovsepyan, M.; Geoghegan, K. F.; Chrunyk, B.A.; Engel, P. C. A Thermally Sensitive Loop in Clostridial Glutamate Dehydrogenase Detected by Limited Proteolysis. J. Biol. Chem. 2003, 278, 1067-1974.

10. Charier, V.; Luba, J.; Parsonage, D.; Claiborne, A. Limited Proteolysis as a Structural Probe of the Soluble $\alpha$-Glycerophosphate Oxidase from Streptococcus sp. Biochemistry 2000, 39, 5035-5044.

11. Kim, Y. J.; Kim, Y. A.; Park, N.; Son, H. S.; Kim, K. S.; Hahn, J. H. Structural Characterization of the Molten Globule State of Apomyoglobin by Limited Proteolysis and HPLC-Mass Spectrometry. Biochemistry 2005, 44, 7490-7496.

12. Hong, H.; Appleyard, A. N.; Siskos, A. P.; Garcia-Bernardo, J.; Staunton, J.; Leadlay, P. F. Chain Initiation on Type I Modular Polyketide Synthases Revealed by Limited Proteolysis and Ion-Trap Mass Spectrometry. FEBS J. 2005, 272, 2373-2387. 
13. Jawhari, A.; Boussert, S.; Lamour, V.; Atkinson, R. A.; Kieffer, B.; Poch, O.; Potier, N. Van, D. A. Moras, D.; Poterszman, A. Domain Architecture of the p62 Subunit from the Human Transcription/Repair Factor TFIIH Deduced by Limited Proteolysis and Mass Spectrometry Analysis. Biochemistry 2004, 43, 14420-14430.

14. Schlosser, A.; Klockow, B.; Manstein, D. J.; Lehmann, W. D. Analysis of Post-translational Modification and Characterization of the Domain Structure of Dynamin A from Dictyostelium Discoideum. J. Mass Spectrom. 2003, 38, 277-282.

15. Wang, L.; Hare, M.; Hays, T. S.; Barbar, E. Dynein Light Chain LC8 Promotes Assembly of the Coiled-Coil Domain of Swallow Protein. Biochemistry 2004, 43, 4611-4620.

16. Heegaard, N. H. H.; Jorgensen, T. J. D.; Rozlosnik, N.; Corlin, D. B.; Pedersen, J. S.; Tempesta, A. G.; Roepstorff, P.; Bauer, R.; Nissen, M. H. Unfolding, Aggregation, and Seeded Amyloid Formation of Lysine-58Cleaved 2-Microglobulin. Biochemistry 2005, 44, 4397-4407.

17. Myers, S. L.; Thomson, N. H.; Radford, S. E.; Ashcroft, A. E. Investigating the Structural Properties of Amyloid-like Fibrils Formed In Vitro from 2-Microglobulin Using Limited Proteolysis and Electrospray Ionisation Mass Spectrometry. Rapid Commun. Mass Spectrom. 2006, 20, 1628-1636.

18. Bothner, B.; Dong, X. F.; Bibbs, L.; Johnson, J. E.; Siuzdak, G. Evidence of Viral Capsid Dynamics Using Limited Proteolysis and Mass Spectrometry. J. Biol. Chem. 1998, 273, 673-676.

19. Gadgil, H. S.; Bondarenko, P. V.; Pipes, G. D.; Dillon, T. M.; Banks, D.; Abel, J.; Kleemann, G. R.; Treuheit, M. J. Identification of Cysteinylation of a Free Cysteine in the Fab Region of a Recombinant Monoclonal IgG1 Antibody Using Lys-C Limited Proteolysis Coupled with LC/MS Analysis. Anal. Biochem. 2006, 355, 165-174.

20. Hicks, L. M.; O'Connor, S. E.; Mazur, M. T.; Walsh, C. T.; Kelleher, N. L. Mass Spectrometric Interrogation of Thioester-bound Intermediates in the Initial Stages of Epothilone Biosynthesis. Chem. Biol. 2004, 11, 327-335.

21. Mazur, M. T.; Walsh, C. T.; Kelleher, N. L. Site-specific Observation of Acyl Intermediate Processing in Thiotemplate Biosynthesis by Fourier Transform Mass Spectrometry: The Polyketide Module of Yersiniabactin Synthetase. Biochemistry 2003, 43, 13393-13400.

22. Leite, J. F.; Amoscato, A. A.; Cascio, M. Coupled Proteolytic and Mass Spectrometry Studies Indicate a Novel Topology for the Glycine Receptor. J. Biol. Chem. 2000, 275, 13683-13689.

23. Pinkse, M. W. H.; Heck, A. J. R.; Rumpel, K.; Pullen, F. Probing Noncovalent Protein-Ligand Interactions of the cGMP-dependent Protein Kinase Using Electrospray Ionization Time of Flight Mass Spectrometry. J. Am. Soc. Mass Spectrom. 2004, 15, 1392-1399.
24. Spolaore, B.; Bermejo, R.; Zambonin, M.; Fontana, A. Protein Interactions Leading to Conformational Changes Monitored by Limited Proteolysis: Apo Form and Fragments of Horse Cytochrome c. Biochemistry 2001, 40, 9460-9468.

25. Huang, S.; Zou, X.; Guo, P.; Zhong, L.; Peng, J.; Jing, G. Probing the Subtle Conformational State of N138ND2-Q106O Hydrogen Bonding Deletion Mutant (Asn138Asp) of Staphylococcal Nuclease Using Time of Flight Mass Spectrometry with Limited Proteolysis. Arch. Biochem. Biophys. 2005, 434, 86-92.

26. Esteban, B.; Carrascal, M.; Abian, J.; Lamparter, T. Light-induced Conformational Changes of Cyanobacterial Phytochrome Cph1 Probed by Limited Proteolysis and Autophosphorylation. Biochemistry 2005, 44, $450-461$.

27. McDonald, C.; Li, L. Limited Proteolysis Combined with Isotope Labeling and Quantitative LC-MALDI MS for Monitoring Protein Conformational Changes: A Study on Calcium-binding Sites of Cardiac Troponin C. Anal. Chim. Acta 2005, 534, 3-10.

28. Koth, C. M.; Orlicky, S. M.; Larson, S. M.; Edwards, A. M. Use of Limited Proteolysis to Identify Protein Domains Suitable for Structural Analysis. Methods Enzymol. 2003, 368, 77-84.

29. Gao, X.; Bain, K.; Bonanno, J. B.; Buchanan, M.; Henderson, D.; Lorimer D.; Marsh, C.; Reynes, J. A.; Sauder, J. M.; Schwinn, K.; Thai, C.; Burley, S. K. High-throughput Limited Proteolysis/Mass Spectrometry for Protein Domain Elucidation. J. Struct. Funct. Genomics 2005, 6, 129-134.

30. Chen, G.; Sun, Y.; Jin, R.; Gouaux, E. Probing the Ligand Binding Domain of the GluR2 Receptor by Proteolysis and Deletion Mutagenesis Defies Domain Boundaries and Yields a Crystallizable Construct. Protein Sci. 1998, 7, 2623-2630.

31. Stroh, J. G.; Loulakis, P.; Lanzetti, A. J.; Xie, J. LC-Mass Spectrometry Analysis of N- and C-Terminal Boundary Sequences of Polypeptide Fragments by Limited Proteolysis. J. Am. Soc. Mass Spectrom. 2005, 16, $38-45$.

32. Meng, W.; Swenson, L. L.; Fitzgibbon, M. J.; Hayakawa, K.; Ter Haar, E.; Behrens, A. E.; Fulghum, J. R.; Lippke, J. A. Structure of Mitogenactivated Protein Kinase-activated Protein (MAPKAP) Kinase 2 Suggests a Bifunctional Switch That Couples Kinase Activation with Nuclear Export. J. Biol. Chem. 2002, 277, 37401-37405.

33. Hicks, L. M.; Mazur, M. T.; Miller, L. M.; Dorrestein, P. C.; Schnarr N. A.; Khosla, C.; Kelleher, N. L. Investigating Nonribosomal Peptide and Polyketide Biosynthesis by Direct Detection of Intermediates on $>70 \mathrm{kDa}$ Polypeptides by Using Fourier-Transform Mass Spectrometry. Chem. Biol. Chem. 2006, 7, 904-907.

34. Horn, D. M.; Zubarev, R. A.; McLafferty, F. W. Automated De Novo Sequencing of Proteins by Tandem High-Resolution Mass Spectrometry. Proc. Natl. Acad. Sci. U.S.A. 2000, 97, 10313-10317. 